\title{
Commentary: Leucine-rich Repeat Receptor-Like Gene Screen Reveals that Nicotiana RXEG1 Regulates Glycoside Hydrolase 12 MAMP Detection
}

\author{
Si Wu, Wei Wang*, Xiangru Xu and Xiaolin Wu \\ State Key Laboratory of Wheat and Maize Crop Science, Collaborative Innovation Center of Henan Grain Crops, College of \\ Sciences, Henan Agricultural University, Zhengzhou, China
}

Keywords: genome-wide VIGS screen, leucine-rich repeat (LRR) receptor-like gene (protein), microbe-associated molecular patterns (MAMPs), Nicotiana benthamiana, pattern-recognition receptors (PRRs), plant immune response, response to XEG1 (RXEG1) protein, virus-induced gene silencing (VIGS)

\section{A Commentary on}

Leucine-rich Repeat Receptor-Like Gene Screen Reveals that Nicotiana RXEG1 Regulates Glycoside Hydrolase 12 MAMP Detection

by Wang, Y., Xu, Y., Sun, Y., Wang, H., Qi, J., Wan, B., et al. (2018). Nat. Commun. 9:594. doi: 10. 1038/s41467-018-03010-8

\section{OPEN ACCESS}

Edited by:

Emmanouil Dermitzakis,

Université de Genève, Switzerland

Reviewed by: Jiannis Ragoussis,

McGill University, Canada

*Correspondence:

Wei Wang

wangwei@henau.edu.cn

Specialty section

This article was submitted to Genomic Assay Technology,

a section of the journal

Frontiers in Genetics

Received: 14 March 2018 Accepted: 14 February 2019

Published: 07 March 2019

Citation:

Wu S, Wang W, Xu X and Wu X (2019) Commentary: Leucine-rich Repeat Receptor-Like Gene Screen Reveals that Nicotiana RXEG1 Regulates Glycoside Hydrolase 12 MAMP Detection. Front. Genet. 10:162. doi: 10.3389/fgene.2019.00162
Innate immunity against pathogen infection by membrane-localized receptors is evolutionarily conserved among eukaryotes (Palma et al., 2007). In plants, innate immunity is essential for defending against harmful microbes that cause dramatic losses in agriculture. Activation of plant immunity is generally determined by pattern-recognition receptors (PRRs). In contrast to mammals, all known plant PRRs are localized at the cell surface (Macho and Zipfel, 2014). There, they detect potentially harmful microbes by recognizing microbe-associated molecular patterns (MAMPs) (Boller and Felix, 2009; Böhm et al., 2014). The diversity and number of MAMPs recognized by individual plant species is likely larger than previously thought (Brunner and Nürnberger, 2012; Zhang et al., 2013; Böhm et al., 2014; Macho et al., 2014). Thus, characterizing PRRs in plants is key to plant-microbe interaction studies.

PRRs such as receptor-like kinases (RLKs) and receptor-like proteins (RLPs) (Shiu et al., 2004; Fischer et al., 2016) play crucial roles in plant immunity, growth, and development. Plant genomes contain hundreds of such receptor-like genes, most of which encode proteins with extracellular leucine-rich repeat (LRR) domains (Shiu and Bleecker, 2003; Shiu et al., 2004; Diévart et al., 2011; Sakamoto et al., 2012), but only a few PRRs with LRR domains have been identified (Sun et al., 2013; Hind et al., 2016; Tang et al., 2017; Wang et al., 2018). Therefore, an efficient approach for quickly identifying PRRs is important for understanding plant innate immunity and developing disease-resistant plants.

Recently, Wang et al. (2018) developed a high-throughput virus-induced gene silencing (VIGS)-based toolkit for characterizing LRR receptor-like genes on a genomic scale (Figure 1A). The approach was demonstrated on the plant model Nicotiana benthamiana, a solanaceous plant and a close relative of tobacco. As a result, 257 tobacco rattle virus-based constructs were generated to silence all 403 identified genes that encode predicted membrane-localized LRR-RLPs and LRR-RLKs in N. benthamiana. Moreover, Wang et al. (2018) successfully identified Response to XEG1 (RXEG1), an LRR-RLP that specifically recognizes the glycoside hydrolase 12 protein XEG1. Their 
study demonstrated that this genome-wide silencing assay can quickly identify new immune receptors to help mine and utilize crop resistance resources. VIGS has also been successfully developed for monocots (Yuan et al., 2011; Liou et al., 2014; Liu et al., 2016). In view of the importance of monocot cereals such as rice, wheat, and maize-and great yield losses due to microbial attacks-it would be worthwhile to evaluate this method for cereal crops.

The VIGS-based approach for identification of PRRs has advantages over methods that rely on map-based cloning and Arabidopsis T-DNA insertion lines (Zipfel et al., 2006; Jehle et al., 2013; Zhang et al., 2013; Albert et al., 2015): (1) VIGS

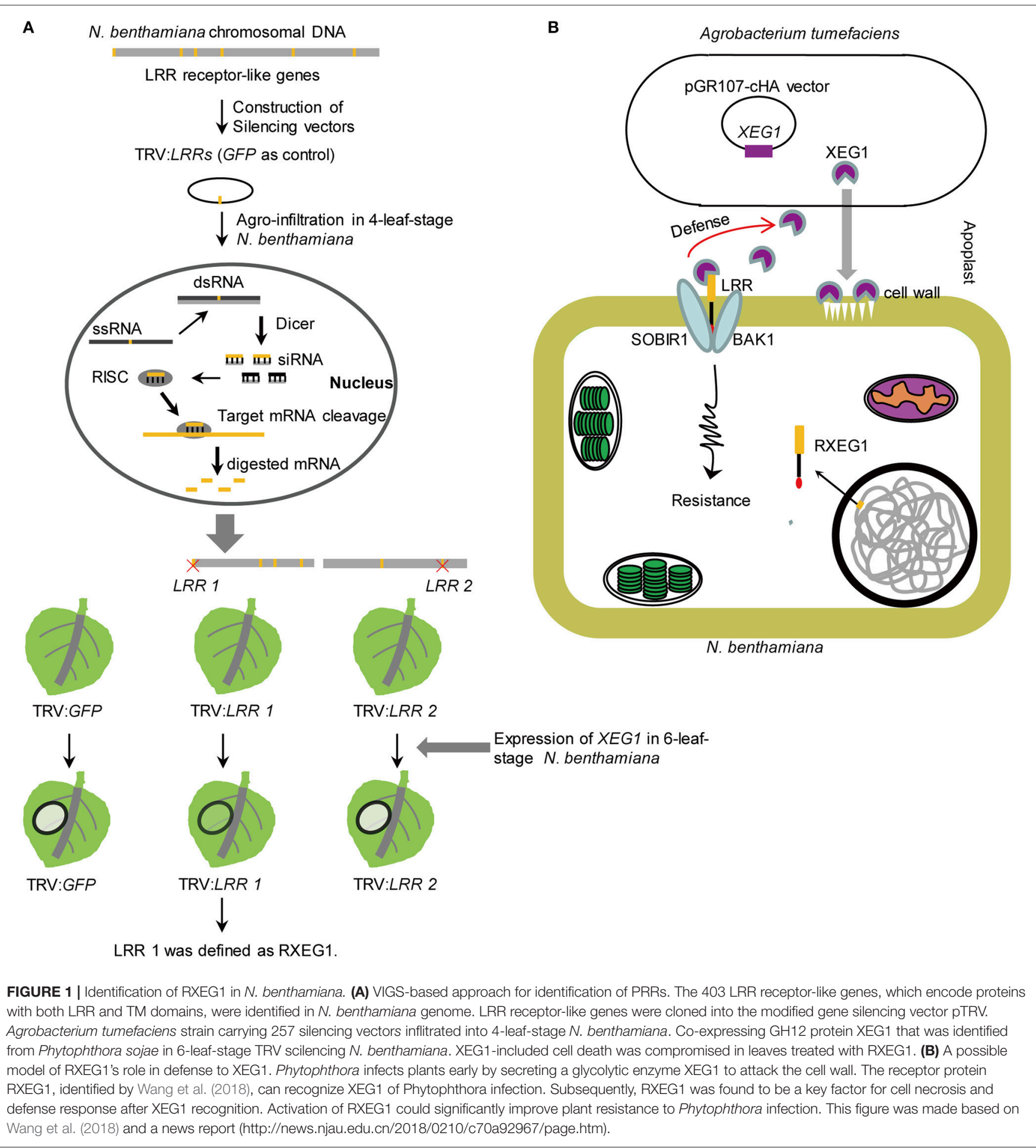


can be easily performed on N. benthamiana, which are amenable to highly efficient VIGS and protein expression (within one month); and (2) VIGS in N. benthamiana avoids gene function redundancy and allows for simultaneous silencing of multiple homologous genes (Wang et al., 2018). Thus, silencing efficiency can be evaluated by analyzing gene expression and alterations in plant growth. The silencing efficiency of 230 LRR receptorlike genes (i.e., 43 LRR-RLP and 187 LRR-RLK genes) is $>60 \%$ (Wang et al., 2018).

In addition to identifying and characterizing PRRs, elucidating the mechanisms by which PRRs perceive microbial attack will significantly advance our understanding of plant innate immunity. The comprehensive and intensive work of Wang et al. (2018) revealed how $N$. benthamiana recognizes XEG1, a widely distributed MAMP in microbial taxa (Figure 1B). When microbes attack plants, XEG1 is secreted early into the infected plants to degrade xyloglucan and $\beta$-glucan in plant cell walls (Ma et al., 2015; Gui et al., 2017). Wang et al. (2018) demonstrated that RXEG1 specifically recognizes XEG1, associates XEG1 via the LRR domain in the apoplast, and forms a complex with the LRR receptor-like kinases BAK1 and SOBIR1 to transduce the XEG1-induced defense signal. The mode of action for RXEG1 will help elucidate the mechanisms of disease resistance and regulation of various crops in the future. RXEG1 in $N$. benthamiana can identify XEG1 family proteins secreted by various microbes. Therefore, RXEG1 could potentially be used to protect a broad range of plants, especially other solanaceous plant species such tomatoes, whereby high disease resistance might be achieved through genetic engineering or by spraying.

\section{REFERENCES}

Albert, I., Böhm, H., Albert, M., Feiler, C. E., Imkampe, J., Wallmeroth, N., et al. (2015). An RLP23-SOBIR1-BAK1 complex mediates NLP-triggered immunity. Nat. Plants 1:15140. doi: 10.1038/nplants.2015.140

Böhm, H., Albert, I., Fan, L., Reinhard, A., and Nürnberger, T. (2014). Immune receptor complexes at the plant cell surface. Curr. Opin. Plant Biol. 20, 47-54. doi: 10.1016/j.pbi.2014.04.007

Boller, T., and Felix, G. (2009). A renaissance of elicitors: perception of microbe-associated molecular patterns and danger signals by pattern-recognition receptors. Аnnu. Rev. Plant Biol. 60, 379-406. doi: 10.1146/annurev.arplant.57.032905.105346

Brunner, F., and Nürnberger, T. (2012). Identification of immunogenic microbial patterns takes the fast lane. Proc. Natl. Acad. Sci. U.S.A. 109, 4029-4030. doi: 10.1073/pnas.1201444109

Diévart, A., Gilbert, N., Droc, G., Attard, A., Gourgues, M., Guiderdoni, E., et al. (2011). Leucine-rich repeat receptor kinases are sporadically distributed in eukaryotic genomes. BMC Evol. Biol. 11:367. doi: 10.1186/1471-2148-11-367

Fischer, I., Diévart, A., Droc, G., Dufayard, J. F., and Chantret, N. (2016). Evolutionary dynamics of the leucine-rich repeat receptor-like kinase (LRR-RLK) subfamily in Angiosperms. Plant Physiol. 170, 1595-1610. doi: 10.1104/pp.15.01470

Franco-Orozco, B., Berepiki, A., Ruiz, O., Gamble, L., Griffe, L. L., Wang, S., et al. (2017). A new proteinaceous pathogen-associated molecular pattern (PAMP) identified in Ascomycete fungi induces cell death in Solanaceae. N. Phytol. 214, 1657-1672. doi: 10.1111/nph.14542

Gui, Y. J., Chen, J. Y., Zhang, D. D., Li, N. Y., Li, T. G., Zhang, W. Q., et al. (2017). Verticillium dahliae manipulates plant immunity by glycoside hydrolase 12 proteins in conjunction with carbohydrate-binding module 1. Environ. Microbiol. 19, 1914-1932. doi: 10.1111/1462-2920.13695
Wang et al. (2018) identified a new PRR (RXEG1) and its associated PKs. Further experimentation is needed to reveal ligand binding specificity, structural requirements, and modifications of RXEG1. More specifically, studies are needed to reveal how dynamics and protein composition are controlled during immune receptor complex assembly, and which intracellular hubs are involved in transforming various microbial signal inputs into a generic plant immune response.

Currently, the recognition of certain MAMPs remains restricted to solanaceous plants (Wang et al., 2016, 2018; Franco-Orozco et al., 2017). For example, RXEG1-like genes or proteins have been found in various dicots, but not in monocots. Silencing LRR receptor candidates for identification of MAMP recognition receptors in cereal crops is a straightforward approach that would advance MAMP recognition and improve crop resistance.

\section{AUTHOR CONTRIBUTIONS}

WW drafted the manuscript. SW and WW revised the manuscript. SW draw Figure 1. All authors contributed to the writing of the manuscript.

\section{ACKNOWLEDGMENTS}

Work in our lab was supported by the National Natural Science Foundation of China (31771700 and 31701343). We thank Dr. Jinghua Zhang and Dr. Hui Liu for valuable discussion in drawing Figure 1.

Hind, S. R., Strickler, S. R., Boyle, P. C., Dunham, D. M., Bao, Z., O’Doherty, I. M., et al. (2016). Tomato receptor FLAGELLIN-SENSING 3 binds flgII-28 and activates the plant immune system. Nat. Plants 2:16128. doi: 10.1038/nplants.2016.128

Jehle, A. K., Lipschis, M., Albert, M., Fallahzadeh-Mamaghani, V., Fürst, U., Mueller, K., et al. (2013). The receptor-like protein ReMAX of Arabidopsis detects the microbe-associated molecular pattern eMax from Xanthomonas. Plant Cell 25, 2330-2340. doi: 10.1105/tpc.113.110833

Liou, M. R., Huang, Y. W., Hu, C. C., Lin, N. S., and Hsu, Y. H. (2014). A dual gene-silencing vector system for monocot and dicot plants. Plant Biotechnol. J. 12, 330-343. doi: 10.1111/pbi.12140

Liu, N., Xie, K., Jia, Q., Zhao, J., Chen, T., Li, H., et al. (2016). Foxtail mosaic virus-induced gene silencing in monocot plants. Plant Physiol. 171, 1801-1807. doi: 10.1104/pp.16.00010

Ma, Z., Song, T., Zhu, L., Ye, W., Wang, Y., Shao, Y., et al. (2015). A Phytophthora sojae glycoside hydrolase 12 protein is a major virulence factor during soybean infection and is recognized as a PAMP. Plant Cell 27, 2057-2072. doi: $10.1105 /$ tpc. 15.00390

Macho, A. P., Schwessinger, B., Ntoukakis, V., Brutus, A., Segonzac, C., Roy, S., et al. (2014). A bacterial tyrosine phosphatase inhibits plant pattern recognition receptor activation. Science 343, 1509-1512. doi: 10.1126/science.1248849

Macho, A. P., and Zipfel, C. (2014). Plant PRRs and the activation of innate immune signaling. Mol. Cell 54, 263-272. doi: 10.1016/j.molcel.2014. 03.028

Palma, K., Zhao, Q., Cheng, Y. T., Bi, D., Monaghan, J., Cheng, W., et al. (2007). Regulation of plant innate immunity by three proteins in a complex conserved across the plant and animal kingdoms. Genes Dev. 21, 1484-1493. doi: $10.1101 /$ gad.1559607

Sakamoto, T., Deguchi, M., Brustolini, O. J., Santos, A. A., Silva, F. F., and Fontes, E. P. (2012). The tomato RLK superfamily: phylogeny and functional 
predictions about the role of the LRRII-RLK subfamily in antiviral defense. BMC Plant Biol. 12:229. doi: 10.1186/1471-2229-12-229

Shiu, S. H., and Bleecker, A. B. (2003). Expansion of the receptor-like kinase/Pelle gene family and receptor-like proteins in Arabidopsis. Plant Physiol. 132, 530-543. doi: 10.1104/pp.103.021964

Shiu, S. H., Karlowski, W. M., Pan, R., Tzeng, Y. H., Mayer, K. F., and $\mathrm{Li}, \mathrm{W}$. H. (2004). Comparative analysis of the receptor-like kinase family in Arabidopsis and rice. Plant Cell 16, 1220-1234. doi: 10.1105/tpc. 020834

Sun, Y., Li, L., Macho, A. P., Han, Z., Hu, Z., Zipfel, C., et al. (2013). Structural basis for flg22-induced activation of the Arabidopsis FLS2-BAK1 immune complex. Science 342, 624-628. doi: 10.1126/science.1243825

Tang, D., Wang, G., and Zhou, J. M. (2017). Receptor kinases in plantpathogen interactions: more than pattern recognition. Plant Cell 29, 618-637. doi: 10.1105/tpc.16.00891

Wang, L., Albert, M., Einig, E., Fürst, U., Krust, D., Felix, G., et al. (2016). The pattern-recognition receptor CORE of Solanaceae detects bacterial cold-shock protein. Nat. Plants 2:16185. doi: 10.1038/nplants.2016.185

Wang, Y., Xu, Y., Sun, Y., Wang, H., Qi, J., Wan, B., et al. (2018). Leucine-rich repeat receptor-like gene screen reveals that Nicotiana RXEG1 regulates glycoside hydrolase 12 MAMP detection. Nat. Commun. 9:594. doi: $10.1038 / \mathrm{s} 41467-018-03010-8$
Yuan, C., Li, C., Yan, L., Jackson, A. O., Liu, Z., Han, C., et al. (2011). A high throughput barley stripe mosaic virus vector for virus induced gene silencing in monocots and dicots. PLoS ONE 6:e26468. doi: 10.1371/journal.pone.0026468

Zhang, W., Fraiture, M., Kolb, D., Löffelhardt, B., Desaki, Y., Boutrot, F. F., et al. (2013). Arabidopsis receptor-like protein 30 and receptor-like kinase suppressor of BIR-1/EVERSGHED mediate innate immunity to necrotrophic fungi. Plant Cell 25, 4227-4241. doi: 10.1105/tpc.113.117010

Zipfel, C., Kunze, G., Chinchilla, D., Caniard, A., Jones, J. D., Boller, T., et al. (2006). Perception of the bacterial PAMP EF-Tu by the receptor EFR restricts Agrobacterium-mediated transformation. Cell 125, 749-760. doi: $10.1016 /$ j.cell.2006.03.037

Conflict of Interest Statement: The authors declare that the research was conducted in the absence of any commercial or financial relationships that could be construed as a potential conflict of interest.

Copyright $\odot 2019 \mathrm{Wu}$, Wang, Xu and Wu. This is an open-access article distributed under the terms of the Creative Commons Attribution License (CC BY). The use, distribution or reproduction in other forums is permitted, provided the original author(s) and the copyright owner(s) are credited and that the original publication in this journal is cited, in accordance with accepted academic practice. No use, distribution or reproduction is permitted which does not comply with these terms. 